\title{
An example on approximation by fat arcs and fat biarcs
}

\author{
Jun-Hai Yong *, Xiao Chen, Jean-Claude Paul \\ Institute of CG and CAD, School of Software, Tsinghua University, Beijing 100084, People's Republic of China
}

Received 21 April 2005; accepted 4 January 2006

\begin{abstract}
Fat arcs form bounding boxes for planar curves. An example on approximation by fat arcs, provided by Qun and Rokne, is corrected. The third derivative of the given curve segment under the polar coordinate system is increasing only at the beginning of the curve segment, and then decreasing, rather than monotonically increasing for the whole curve segment. Some numerical data are corrected as well.
\end{abstract}

(C) 2006 Elsevier Ltd. All rights reserved.

Keywords: Circular arc; Fat arc; Biarc

Circular arcs play a very important role in CAD (ComputerAided Design) and CNC (Computerized Numerical Control) [1,4,5 et al.]. Currently, fat arcs are used to define bounding boxes for planar curves [2,3]. In [2], an example is given to illustrate the approximation algorithm by fat arcs. Here, the example is corrected.

In the example, the given curve is

$t(\alpha)=\mathrm{e}^{0.2(2 \pi+\alpha)}$

under a polar coordinate system $(t, \alpha)$. Under the Cartesian coordinate system with the same center point as the polar coordinate system and with the $x$-axis being the polar axis, $t(\alpha)$ becomes

$\mathbf{S}(\alpha)=\left(\mathbf{S}_{x}(\alpha), \mathbf{S}_{y}(\alpha)\right)=\left(\mathrm{e}^{0.2(2 \pi+\alpha)} \cos (\alpha), \mathrm{e}^{0.2(2 \pi+\alpha)} \sin (\alpha)\right)$,

where the notations $\mathbf{S}_{x}(\alpha)$ and $\mathbf{S}_{y}(\alpha)$ follow the rule, in [2], that uses the symbols ' $\mathbf{P}_{x}$ ' and ' $\mathbf{P}_{y}$ ' to represent the $x$ and $y$ components, respectively, of an arbitrary point (or curve) $\mathbf{P}$. The rule is followed below as well. Let $\alpha_{-1}=-\pi / 8, \alpha_{0}=0$ and $\alpha_{1}=\pi / 8$. We obtain three points

$\mathbf{P}_{-1}=\mathbf{S}\left(\alpha_{-1}\right)=(3.001,-1.243)$,

$\mathbf{P}_{0}=\mathbf{S}\left(\alpha_{0}\right)=(3.514,0), \quad \mathbf{P}_{1}=\mathbf{S}\left(\alpha_{1}\right)=(3.511,1.454)$,

and three distances

\footnotetext{
* Corresponding author. Tel.: +8610 62795455; fax: +861062795460.

E-mail address: yonghh@tsinghua.edu.cn (J.-H. Yong).
}

$l_{0}=\left\|\mathbf{P}_{-1}-\mathbf{P}_{0}\right\|_{2}=1.345, \quad l_{1}=\left\|\mathbf{P}_{1}-\mathbf{P}_{0}\right\|_{2}=1.454$,

$l=\left\|\mathbf{P}_{-1}-\mathbf{P}_{1}\right\|_{2}=2.745$.

Here, the numerical values of $\mathbf{P}_{1}$ and $l$ are different from those in [2], which gives:

$\mathbf{P}_{1}=(5.511,1.454)$ and $l=2.645$.

The unsigned angle between the $x$-axis and the line segment $\mathbf{P}_{-1} \mathbf{P}_{0}$ is

$\beta_{-1}=\operatorname{tg}^{-1}\left(\left|\frac{\left(\mathbf{P}_{0}\right)_{y}-\left(\mathbf{P}_{-1}\right)_{y}}{\left(\mathbf{P}_{0}\right)_{x}-\left(\mathbf{P}_{-1}\right)_{x}}\right|\right)=1.18$,

and the unsigned angle between the $x$-axis and the line segment $\mathbf{P}_{1} \mathbf{P}_{0}$ is

$\beta_{0}=\operatorname{tg}^{-1}\left(\left|\frac{\left(\mathbf{P}_{0}\right)_{y}-\left(\mathbf{P}_{1}\right)_{y}}{\left(\mathbf{P}_{0}\right)_{x}-\left(\mathbf{P}_{1}\right)_{x}}\right|\right)=1.57$,

where $\operatorname{tg}^{-1}(\cdot)$ represents the inverse function of $\operatorname{tg}(\cdot)=\sin (\cdot) /$ $\cos (\cdot)$. Thus, we obtain:

$\varphi_{0}=\pi-\beta_{-1}-\beta_{0}=0.393$.

Ref. [2] provides the wrong formulae for $\beta_{-1}, \beta_{0}$ and $\varphi_{0}$.

The points $\mathbf{P}_{-1}, \mathbf{P}_{0}$ and $\mathbf{P}_{1}$ determine a unique circle with the center point

$\mathbf{O}=\left(-8.604 \times 10^{-15}, 0.722\right)$

and the radius $r_{0}=\left\|\mathbf{P}_{-1}-\mathbf{O}\right\|_{2}=3.587$ (see the dashed circle in Fig. 1). Fig. 1(b) gives a magnified view of Fig. 1(a). In Fig. 1, the solid curve from $\mathbf{P}_{-1}$ to $\mathbf{P}_{1}$ is the given curve $t(\alpha)$ or $\mathbf{S}(\alpha)$ under the Cartesian coordinate system. It is very close to the dashed circle as shown in Fig. 1. 

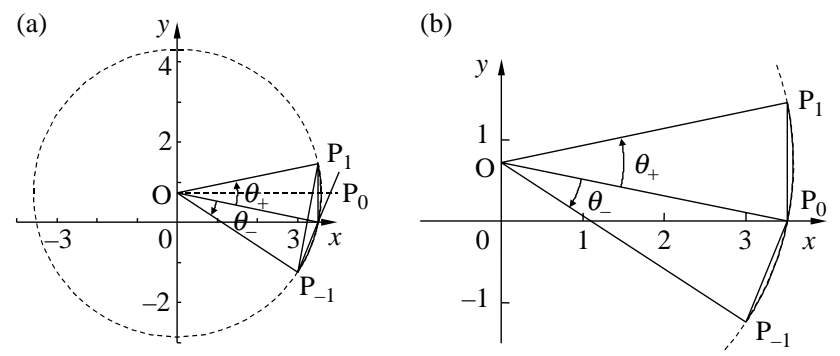

Fig. 1. The given curve $t(\alpha)$ (solid) and the circle (dashed).

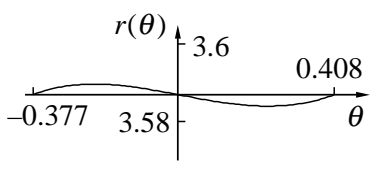

(a) $r(\theta)$

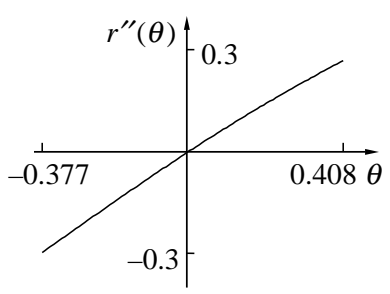

(c) $r^{\prime \prime}(\theta)$

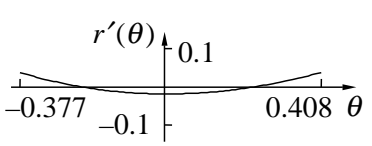

(b) $r^{\prime}(\theta)$

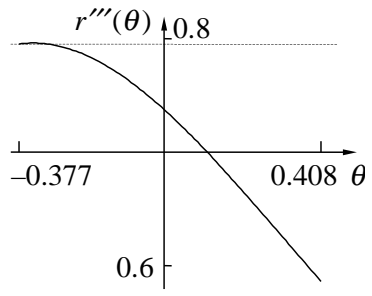

(d) $r^{\prime \prime \prime}(\theta)$
Fig. 2. $r(\theta)$ and its first three derivatives.

Let

$\theta_{-1}=\operatorname{tg}^{-1}\left(\left|\frac{\left(\mathbf{P}_{-1}\right)_{y}-\mathbf{O}_{y}}{\left(\mathbf{P}_{-1}\right)_{x}-\mathbf{O}_{x}}\right|\right)=0.580$

$\theta_{0}=\operatorname{tg}^{-1}\left(\left|\frac{\left(\mathbf{P}_{0}\right)_{y}-\mathbf{O}_{y}}{\left(\mathbf{P}_{0}\right)_{x}-\mathbf{O}_{x}}\right|\right)=0.203$

and

$\theta_{1}=\operatorname{tg}^{-1}\left(\left|\frac{\left(\mathbf{P}_{1}\right)_{y}-\mathbf{O}_{y}}{\left(\mathbf{P}_{1}\right)_{x}-\mathbf{O}_{x}}\right|\right)=0.206$

be the unsigned angles between the $x$-axis and the line segments $\mathbf{O P} \mathbf{P}_{-1}, \mathbf{O} \mathbf{P}_{0}$ and $\mathbf{O} \mathbf{P}_{1}$, respectively.
We build a new polar coordinate system with the center point being $\mathbf{O}$ and the pole axis being the ray starting from $\mathbf{O}$ and passing through the point $\mathbf{P}_{0}$. Under the new polar coordinate system, the curve $t(\alpha)$ becomes $r(\theta)$, where the polar radius is equal to the distance from $t(\alpha)$ to the new center point O, i.e.

$$
r(\alpha)=\sqrt{\left(\mathrm{e}^{0.2(2 \pi+\alpha)} \cos (\alpha)-\mathbf{O}_{x}\right)^{2}+\left(\mathrm{e}^{0.2(2 \pi+\alpha)} \sin (\alpha)-\mathbf{O}_{y}\right)^{2}},
$$

and the polar angle is:

$\theta(\alpha)=\operatorname{tg}^{-1}\left(\frac{\mathrm{e}^{0.2(2 \pi+\alpha)} \sin (\alpha)-\mathbf{O}_{y}}{\mathrm{e}^{0.2(2 \pi+\alpha)} \cos (\alpha)-\mathbf{O}_{x}}\right)+\theta_{0}$.

Let

$\theta_{-}=\theta_{-1}-\theta_{0}=0.377$ and $\theta_{+}=\theta_{0}+\theta_{1}=0.408$.

Then the parametric domain of the curve segment $r(\theta)$ from $\mathbf{P}_{-1}$ to $\mathbf{P}_{1}$ is:

$\Theta=\left[-\theta_{-}, \theta_{+}\right]=[-0.377,0.408]$.

Thus, we have:

$\left(\Theta+\theta_{-}\right) \Theta\left(\Theta-\theta_{+}\right)=[-0.252,0.233]$.

For convenience, let $r^{\prime}(\theta), r^{\prime \prime}(\theta)$ and $r^{\prime \prime \prime}(\theta)$ be the first, the second and the third derivatives of $r(\theta)$ with respect to $\theta$, respectively. Similarly, let $r^{\prime}(\alpha), r^{\prime \prime}(\alpha)$ and $r^{\prime \prime \prime}(\alpha)$ be the first, the second and the third derivatives of $r(\alpha)$ with respect to $\alpha$, respectively, and let $\theta^{\prime}(\alpha), \theta^{\prime \prime}(\alpha)$ and $\theta^{\prime \prime \prime}(\alpha)$ be the first, the second and the third derivatives of $\theta(\alpha)$ with respect to $\alpha$, respectively. We have

$r^{\prime}(\theta)=\frac{r^{\prime}(\alpha)}{\theta^{\prime}(\alpha)}$,

$r^{\prime \prime}(\theta)=\frac{r^{\prime \prime}(\alpha)}{\left(\theta^{\prime}(\alpha)\right)^{2}}-\frac{r^{\prime}(\alpha) \theta^{\prime \prime}(\alpha)}{\left(\theta^{\prime}(\alpha)\right)^{3}}$,

and

Table 1

Comparison of the new results with the results in [1]

\begin{tabular}{|c|c|c|}
\hline Lines & Results in [2] & New results \\
\hline 4 & $\mathbf{P}_{1}=(5.511,1.454)$ & $\mathbf{P}_{1}=(3.511,1.454)$ \\
\hline 6 & $l=2.645$ & $l=2.745$ \\
\hline 7 & $\beta_{-1}=\frac{\left(\mathbf{P}_{-1}\right)_{y}}{\left(\mathbf{P}_{0}\right)_{x}-\left(\mathbf{P}_{-1}\right)_{x}}=1.57$ & $\beta_{-1}=\operatorname{tg}^{-1}\left(\left|\frac{\left(\mathbf{P}_{0}\right)_{y}-\left(\mathbf{P}_{-1}\right)_{y}}{\left(\mathbf{P}_{0}\right)_{x}-\left(\mathbf{P}_{-1} x_{x}\right.}\right|\right)=1.18$ \\
\hline 8 & $\beta_{0}=\frac{\left(\mathbf{P}_{-1}\right)_{y}}{\left(\mathbf{P}_{0}\right)_{x}-\left(\mathbf{P}_{1}\right)_{x}}=1.18$ & $\beta_{0}=\operatorname{tg}^{-1}\left(\left|\frac{\left(\mathbf{P}_{0}\right)_{y}-\left(\mathbf{P}_{1}\right)_{y}}{\left(\mathbf{P}_{0}\right)_{x}-\left(\mathbf{P}_{1}\right)_{x}}\right|\right)=1.57$ \\
\hline 9 & $\varphi_{0}=\pi-\beta_{0}-\beta_{1}=0.393$ & $\varphi_{0}=\pi-\beta_{0}-\beta_{-1}=0.393$ \\
\hline 14 & $R^{\prime \prime \prime}(\Theta)=\left[\min _{-\theta_{-} \leq \theta \leq \theta_{+}} r(\theta), \max _{-\theta_{-} \leq \theta \leq \theta_{+}} r(\theta)\right]$ & $R^{\prime \prime \prime}(\Theta)=\left[\min _{-\theta_{-} \leq \theta \leq \theta_{+}} r^{\prime \prime \prime}(\theta), \max _{-\theta_{-} \leq \theta \leq \theta_{+}} r^{\prime \prime \prime}(\theta)\right]$ \\
\hline 15 & $r^{\prime \prime \prime}$ is monotonically increasing & $\begin{array}{l}r^{\prime \prime \prime}(\theta) \text { is increasing at the beginning, and then decreasing for the } \\
\text { rest part of the curve }\end{array}$ \\
\hline 16 & $R^{\prime \prime \prime}(\Theta) \subseteq[0.00434,0.00509]$ & $R^{\prime \prime \prime}(\Theta)=[0.586,0.796]$ \\
\hline 18 & $\left(\Theta+\theta_{-}\right) \Theta\left(\Theta-\theta_{+}\right)=[0.251,0.233]$ & $\left(\Theta+\theta_{-}\right) \Theta\left(\Theta-\theta_{+}\right)=[-0.252,0.233]$ \\
\hline 20 & {$\left[r_{0 L}, r_{O R}\right]=[3.585,3.589]$} & {$\left[r_{0 L}, r_{O R}\right]=[3.554,3.618]$} \\
\hline $22-23$ & A fat curve of width $3.589-3.585=0.004$ & A fat curve of width $3.618-3.554=0.064$ \\
\hline
\end{tabular}




$$
\begin{aligned}
r^{\prime \prime \prime}(\theta)= & \frac{r^{\prime \prime \prime}(\alpha)}{\left(\theta^{\prime}(\alpha)\right)^{3}}-\frac{3 r^{\prime \prime}(\alpha) \theta^{\prime \prime}(\alpha)}{\left(\theta^{\prime}(\alpha)\right)^{4}}-\frac{r^{\prime}(\alpha) \theta^{\prime \prime \prime}(\alpha)}{\left(\theta^{\prime}(\alpha)\right)^{4}} \\
& +\frac{3 r^{\prime}(\alpha)\left(\theta^{\prime \prime}(\alpha)\right)^{2}}{\left(\theta^{\prime}(\alpha)\right)^{5}},
\end{aligned}
$$

which are shown in Fig. 2(b-d), respectively.

As shown in Fig. 2(d), $r^{\prime \prime \prime}(\theta)$ is increasing only at the beginning, and then monotonically decreasing for the rest part of the curve, rather than monotonically increasing for the whole curve segment as declared in [2]. Hence, the range of $r^{\prime \prime \prime}(\theta)$ from $\mathbf{P}_{-1}$ to $\mathbf{P}_{1}$ is:

$R^{\prime \prime \prime}(\Theta)=[0.586,0.796]$.

Therefore, the radius range of the fat arc is

$$
\begin{aligned}
{\left[r_{0 L}, r_{0 R}\right] } & =r_{0}+\frac{1}{3 !} R^{\prime \prime \prime}(\Theta)\left(\Theta+\theta_{-}\right) \Theta\left(\Theta-\theta_{+}\right) \\
& =[3.554,3.618]
\end{aligned}
$$

according to Formula (11) in [2], and the width of the radius range is $3.618-3.554=0.064$ rather than 0.004 given by [2] The comparison of the above results with those in [2] is summarized in Table 1. In Table 1, the numbers in the 'Lines' column are the line numbers of the corresponding results in the left column of Page 971 of [2].

\section{Acknowledgements}

The research was supported by Chinese 973 Program (2004CB719400) and the National Science Foundation of China $(60403047,60533070)$. The first author was supported by the project sponsored by a Foundation for the Author of National Excellent Doctoral Dissertation of PR China (200342), and a Program for New Century Excellent Talents in University (NCET-04-0088).

\section{References}

[1] Chen X-D, Yong J-H, Zheng G-Q, Sun J-G. Automatic $\mathrm{G}^{1}$ arc spline interpolation for closed point set. Comput Aided Des 2004;36(12): 1205-18.

[2] Lin Q, Rodne JG. Approximation by fat arcs and fat biarcs. Comput Aided Des 2002;34(13):969-79.
[3] Sederberg TW, White SC, Zundel AK. Fat arcs: a bounding region with cubic convergence. Comput Aided Geom Des 1989;6(3):205-18.

[4] Yong J-H, Hu S-M, Sun J-G. A note on approximation of discrete data by $\mathrm{G}^{1}$ arc splines. Comput Aided Des 1999;31(14):911-5.

[5] Yong J-H, Hu S-M, Sun J-G. Bisection algorithms for approximating quadratic Bézier curves by $\mathrm{G}^{1}$ arc splines. Comput Aided Des 2000;32(4): 253-60.

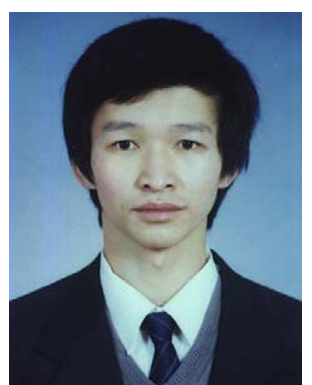

Jun-Hai Yong is a faculty member in School of Software at Tsinghua University, China, since 2002. He received his $\mathrm{BS}$ and $\mathrm{PhD}$ in Computer Science from the Tsinghua University, China, in 1996 and 2001, respectively. He held a visiting researcher position in the Department of Computer Science at Hong Kong University of Science and Technology in 2000. He was a post doctoral fellow in the Department of Computer Science at the University of Kentucky from 2000 to 2002. His research interests include computer-aided design, computer graphics, computer animation, and software engineering.

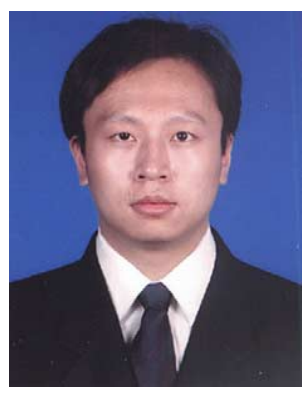

Xiao Chen is a master student in the Department of Computer Science and Technology at Tsinghua University, China. His research interests are computer-aided design and computer graphics.

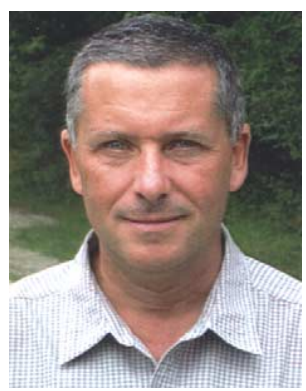

Jean-Claude Paul is a senior researcher at CNRS and INRIA (France), and currently visiting professor at Tsinghua University. He received his $\mathrm{PhD}$ in Mathematics from Paris University in 1976. His research interests include Numerical Analysis, Physics-Based Modeling and ComputerAided Design. 